\title{
Application of the Homotopy Analysis Method for Solving the Variable Coefficient KdV-Burgers Equation
}

\author{
Dianchen Lu and Jie Liu \\ Faculty of Science, Jiangsu University, Zhenjiang, Jiangsu 212013, China \\ Correspondence should be addressed to Dianchen Lu; dclu@ujs.edu.cn
}

Received 22 January 2014; Accepted 21 February 2014; Published 27 March 2014

Academic Editor: Baojian Hong

Copyright ( 2014 D. Lu and J. Liu. This is an open access article distributed under the Creative Commons Attribution License, which permits unrestricted use, distribution, and reproduction in any medium, provided the original work is properly cited.

\begin{abstract}
The homotopy analysis method is applied to solve the variable coefficient KdV-Burgers equation. With the aid of generalized elliptic method and Fourier's transform method, the approximate solutions of double periodic form are obtained. These solutions may be degenerated into the approximate solutions of hyperbolic function form and the approximate solutions of trigonometric function form in the limit cases. The results indicate that this method is efficient for the nonlinear models with the dissipative terms and variable coefficients.
\end{abstract}

\section{Introduction}

To solve the nonlinear partial differential equation (NPDE) has been an attractive research topic for mathematicians and physicists. Nonlinear evolution equations with variable coefficients can describe the physical phenomenon more accurately, and it is of great significance to study how to find the solutions of nonlinear evolution equations with variable coefficients. The nonlinear partial differential equations are generally difficult to solve and their exact solutions are difficult to obtain. In recent years, some various approximate methods have been developed such as homotopy analysis method [1-13] and Adomian's decomposition method [1417] to solve linear and nonlinear differential equations. However, the above works only studied the solutions of equations with constant coefficients. In this work, we apply the homotopy analysis method to the variable coefficients KdV-Burgers equations and obtain the approximate solution of the Jacobi elliptic function form. This method has the merits of simplicity and easy execution.

This paper is arranged in the following manner. In Section 2, we present the homotopy analysis method. In Section 3, the homotopy analysis method on the variable coefficient KdV-Burgers equation is presented. Finally, some conclusions are given.

\section{Basic Idea of Homotopy Analysis Method}

To explain the basic idea of the homotopy analysis method, we consider the following nonlinear differential equation:

$$
A(u)-f(r)=0, \quad r \in \Omega,
$$

subject to boundary condition

$$
B\left(u, \frac{\partial u}{\partial n}\right)=0, \quad r \in \Gamma,
$$

where $A$ is the general differential operator, $B$ is the boundary operator, $f(r)$ is the known analytic function, and $\Gamma$ is the boundary of the region $\Omega$.

Generally speaking, the operator $A$ can be decomposed into linear part $L$ and nonlinear part $N$. Equation (2) therefore can be written as

$$
L(u)+N(u)-f(r)=0 .
$$

Now we set up homotopy mapping $H(u, p): \Omega \times[0,1] \rightarrow R$, which satisfies

$$
H(u, p)=L(u)-L(v)+p(L(v)+N(u)-f(r)),
$$

where $p$ is parameter, $v$ is auxiliary function, and $L(v)+$ $N(v)=0$. 
By (4), we obtain

$$
\begin{gathered}
H(u, 0)=L(u)-L(v), \\
H(u, 1)=A(u)-f(r)=0 .
\end{gathered}
$$

As can be seen from 0 to 1 of $p$ is the process of $L(u)-L(v)$ to $A(u)-f(r)$ of $H(u, p)$; this is the homotopy deformation.

Assume that the solution of $H(u, p)=0$ can be written as a power series in $p$ :

$$
\tilde{u}(x, t, p)=\sum_{i=0}^{\infty} u_{i}(x, t) p^{i}=u_{0}+p u_{1}+p^{2} u_{2}+\cdots
$$

So when $p=0, \tilde{u}(x, t, 0)=u_{0}(x, t)$ is the solution of $L(u)-$ $L(v)=0$; when $p \rightarrow 1$, the approximate solution of $A(u)-$ $f(r)=0$ is $u(x, t)=u_{0}+u_{1}+u_{2}+\cdots$.

\section{Application}

In this section, we focus on the variable coefficient $\mathrm{KdV}$ Burgers equation

$$
u_{t}+u u_{x}+\alpha(t) u_{x x}+\beta(t) u_{x x x}=0,
$$

where $\alpha(t)$ and $\beta(t)$ are any function about $t$.

It is fascinating to observe that, when $\alpha(t)$ and $\beta(t)$ are constant, (7) becomes the well-known KdV-Burgers equation; the equation plays an important role in studying liquid with bubbles inside, the flow of liquid in elastic tubes, and the problems of turbulence [18-20]. When $\alpha(t)$ is constant, $\beta(t)=0$, (7) becomes the well-known Burgers equation. When $\beta(t)$ is constant, $\alpha(t)=0$, (7) becomes the well-known $\mathrm{KdV}$ equation. When $\beta(t)=\beta$ is constant, (7) becomes

$$
u_{t}+u u_{x}+\alpha(t) u_{x x}+\beta u_{x x x}=0 .
$$

Next, we applied the homotopy analysis method to study the approximate solution of (8).

In order to get the solution of (8), we lead in homotopy mapping.

To aim at (8), we set up homotopy mapping $H(u, p): R \times$ $I \rightarrow R$

$$
H(u, p)=L(u)-L(v)+p\left(L(v)+u u_{x}+\alpha(t) u_{x x}\right),
$$

where $R=(-\infty,+\infty), I=[0,1], v$ is the auxiliary function, and the linear operator $L$ is expressed as $L(u)=u_{t}+\beta u_{x x x}$.

By using the generalized elliptic method [21], we can get that the typical $\mathrm{KdV}$ equation corresponding to (8),

$$
v_{t}+v v_{x}+\beta v_{x x x}=0
$$

has the following elliptic function solution:

$$
\begin{aligned}
v_{1}(x, t)= & c_{0}-\beta \frac{6 k^{2} m^{2} s n(\xi, m)+12 k^{2}\left(m^{2}-1\right) c n(\xi, m)}{s n(\xi, m)+c n(\xi, m)+d n(\xi, m)} \\
& +\beta \frac{3 k^{2} m^{4} s n^{2}(\xi, m)-12 k^{2} c n^{2}(\xi, m)}{(\operatorname{sn}(\xi, m)+c n(\xi, m)+d n(\xi, m))^{2}} .
\end{aligned}
$$

When $m \rightarrow 1, v_{1}(x, t)$ degenerates to the following solitary wave solution:

$$
\begin{aligned}
v_{1.1}(x, t)= & c_{0}-\beta \frac{6 k^{2} \tanh \xi}{\tanh \xi+\operatorname{sech} \xi+\operatorname{sech} \xi} \\
& +\beta \frac{3 k^{2} \tanh ^{2} \xi-12 k^{2} \operatorname{sech}^{2} \xi}{(\tanh \xi+\operatorname{sech} \xi+\operatorname{sech} \xi)^{2}} .
\end{aligned}
$$

When $m \rightarrow 0, v_{1}(x, t)$ degenerates to the trigonometric function solution

$$
v_{1.2}(x, t)=c_{0}+\frac{12 k^{2} \beta \cos \xi}{\sin \xi+\cos \xi+1}-\frac{12 k^{2} \beta \cos ^{2} \xi}{(\sin \xi+\cos \xi+1)^{2}},
$$

where $\xi=k x+\left[-c_{0} k+\beta k^{3}\left(4 m^{2}-5\right)\right] t+\xi_{0}, k, c_{0}$, and $\xi_{0}$ are any constant, $m$ is the module, and $0 \leq m \leq 1$.

One can easily prove that $H(u, 1)=0$ and $(8)$ is the same, so the solution $u(x, t)$ of $(8)$ is the solution of $H(u, p)=0$ when under the condition $p \rightarrow 1$.

Let

$$
\widetilde{u}(x, t, p)=\sum_{i=0}^{\infty} u_{i}(x, t) p^{i}=u_{0}+p u_{1}+p^{2} u_{2}+\cdots
$$

be the solution of $H(u, p)=0$; by [22] we can know that this series is uniformly convergent in the $p \in[0,1]$. Thus, it yields that

$$
u=\sum_{i=0}^{\infty} u_{i}(x, t)=u_{0}+u_{1}+u_{2}+\cdots
$$

In order to obtain the approximate solution of (8), we substitute (14) into the equation $H(u, p)=0$. By taking the auxiliary function $v=v_{1}(x, t)$, and comparing the coefficients of the same power of $p$, one can obtain that

$$
\begin{aligned}
& p^{0}: L\left(u_{0}\right)=L(v)=L\left(v_{1}\right) \\
& p^{1}: L\left(u_{1}\right)=-L\left(v_{1}\right)-u_{0} u_{0 x}-\alpha(t) u_{0 x x}=-\alpha(t) u_{0 x x}
\end{aligned}
$$

$$
p^{2}: L\left(u_{2}\right)=-u_{0} u_{1 x}-u_{1} u_{0 x}-\alpha(t) u_{1 x x}
$$

From (16) we have

$$
u_{0}(x, t)=v_{1}(x, t) .
$$

By using the Fourier transform, one can obtain the solution of (17) with the initial condition $\left.u_{1}\right|_{t=0}=0$ as follows:

$$
\begin{aligned}
u_{1}(x, t)=-\frac{1}{2 \pi} \int_{0}^{t} \iint_{-\infty}^{+\infty} \alpha(\tau) v_{1 \xi \xi} & \\
& \times \cos \left[-\lambda^{3} \beta(t-\tau)\right.
\end{aligned}
$$


Similarly, one also finds the solution of (18) with the initial condition $\left.u_{2}\right|_{t=0}=0$ as

$$
\begin{array}{r}
u_{2}(x, t)=\frac{1}{2 \pi} \int_{0}^{t} \int_{-\infty}^{+\infty}\left(-v_{1} u_{1 \xi}-u_{1} v_{1 \xi}-\alpha(t) u_{1 \xi \xi}\right) \\
\times \int_{-\infty}^{+\infty} \cos \left[-\lambda^{3} \beta(t-\tau)\right. \\
+\lambda(x-\xi)] d x d \xi d \tau .
\end{array}
$$

From (11), (12), (13), (20), and (21) the two-degree approximate solution of (8) can be obtained as follows:

$$
\begin{gathered}
u_{2}^{*}(x, t) \\
=c_{0}-\beta \frac{6 k^{2} m^{2} s n(\xi, m)+12 k^{2}\left(m^{2}-1\right) c n(\xi, m)}{s n(\xi, m)+c n(\xi, m)+d n(\xi, m)} \\
+\beta \frac{3 k^{2} m^{4} s n^{2}(\xi, m)-12 k^{2} c n^{2}(\xi, m)}{(s n(\xi, m)+c n(\xi, m)+d n(\xi, m))^{2}} \\
+\frac{1}{2 \pi} \int_{0}^{t} \iint_{-\infty}^{+\infty}\left(-\alpha(\tau) v_{1 \xi \xi}-v_{1} u_{1 \xi}\right. \\
\left.-u_{1} v_{1 \xi}-\alpha(\tau) u_{1 \xi \xi}\right) \\
\times \cos \left[-\lambda^{3} \beta(t-\tau)\right. \\
+\lambda(x-\xi)] d x d \xi d \tau,
\end{gathered}
$$

where $\xi=k x+\left[-c_{0} k+\beta k^{3}\left(4 m^{2}-5\right)\right] t+\xi_{0}, k, c_{0}$, and $\xi_{0}$ are any constant, $m$ is the module, and $0 \leq m \leq 1$. Consider

$$
\begin{array}{r}
v_{0}=c_{0}-\beta \frac{6 k^{2} m^{2} s n(\xi, m)+12 k^{2}\left(m^{2}-1\right) c n(\xi, m)}{s n(\xi, m)+c n(\xi, m)+d n(\xi, m)} \\
+\beta \frac{3 k^{2} m^{4} s n^{2}(\xi, m)-12 k^{2} c n^{2}(\xi, m)}{(s n(\xi, m)+c n(\xi, m)+d n(\xi, m))^{2}}, \\
u_{1}(x, t) \\
=-\frac{1}{2 \pi} \int_{0}^{t} \iint_{-\infty}^{+\infty} \alpha(\tau) v_{1 \xi \xi} \\
\times \cos \left[-\lambda^{3} \beta(t-\tau)\right. \\
+\lambda(x-\xi)] d x d \xi d \tau .
\end{array}
$$

When $m \rightarrow 1$ and $m \rightarrow 0, u_{2}^{*}(x, t)$ degenerates to the following approximate solutions:

$$
\begin{array}{r}
u_{2.1}^{*}(x, t) \\
=c_{0}-\frac{6 \beta k^{2} \tanh \xi}{\tanh \xi+\operatorname{sech} \xi+\operatorname{sech} \xi} \\
+\beta \frac{3 k^{2} \tanh ^{2} \xi-12 k^{2} \operatorname{sech}^{2} \xi}{(\tanh \xi+\operatorname{sech} \xi+\operatorname{sech} \xi)^{2}}
\end{array}
$$

$$
\begin{aligned}
-\frac{1}{2 \pi} \int_{0}^{t} \iint_{-\infty}^{+\infty}( & \alpha(\tau) u_{1 \xi \xi}+v_{1} u_{1 \xi} \\
& \left.+u_{1} v_{1 \xi}+\alpha(\tau) u_{1 \xi \xi}\right) \\
& \times \cos \left[-\lambda^{3} \beta(t-\tau)\right. \\
& +\lambda(x-\xi)] d x d \xi d \tau
\end{aligned}
$$$$
u_{2.2}^{*}(x, t)
$$

$$
\begin{gathered}
=c_{0}+\beta \frac{-6 k^{2} \sin \xi+12 k^{2} \cos \xi}{\sin \xi+\cos \xi+1} \\
-\frac{12 k^{2} \beta \cos ^{2} \xi}{(\sin \xi+\cos \xi+1)^{2}} \\
-\frac{1}{2 \pi} \int_{0}^{t} \iint_{-\infty}^{+\infty} \cos \left[-\lambda^{3} \beta(t-\tau)+\lambda(x-\xi)\right] \\
\quad \times\left(\alpha(\tau) v_{1 \xi}+v_{1} u_{1 \xi}\right. \\
\left.\quad+u_{1} v_{1 \xi}+\alpha(\tau) u_{1 \xi \xi}\right) d x d \xi d \tau .
\end{gathered}
$$

By comparing the higher power coefficients of $p$, more higher power approximate solutions of (8) can also be obtained.

\section{Conclusion}

This work studies the variable coefficients KdV-Burgers equations by using the homotopy analysis method, and the two-degree approximate solution of the Jacobi elliptic function form is obtained, which can degenerate to solitary wave approximate solution and trigonometric function approximate solution in the limit case. Our results show that the homotopy analysis method is applicable to the variable solution equations; how to apply this method to high-degree and high-dimensional system remains to be further studied.

\section{Conflict of Interests}

The authors declare that there is no conflict of interests regarding the publication of this paper.

\section{Acknowledgments}

This work was supported by the National Nature Science Foundation of China (no. 61070231), the Outstanding Personal Program in Six Fields of Jiangsu Province, China (Grant no. 2009188), and the Graduate Student Innovation Project of Jiangsu Province (Grant no. CXLX13_673).

\section{References}

[1] J. H. He, "A simple perturbation approach to Blasius equation," Applied Mathematics and Computation, vol. 140, no. 2-3, pp. 217-222, 2003. 
[2] J. H. He, "Application of homotopy perturbation method to nonlinear wave equations," Chaos, Solitons and Fractals, vol. 26, no. 3, pp. 695-700, 2005.

[3] J. H. He, "Homotopy perturbation method for bifurcation of nonlinear problems," International Journal of Nonlinear Sciences and Numerical Simulation, vol. 6, no. 2, pp. 207-208, 2005.

[4] H. S. Fu, L. Cao, and B. Han, "A homotopy perturbation method for well log constrained seismic waveform inversion," Chinese Journal of Geophysics, vol. 55, no. 9, pp. 2173-2179, 2004.

[5] L. F. Shi and X. C. Zhou, "Homotopic mapping solution of soliton for a class of disturbed Burgers equation," Acta Physica Sinica, vol. 59, no. 5, pp. 2915-2918, 2010.

[6] D. D. Ganji and A. Sadighi, "Application of He's homotopyperturbation method to nonlinear coupled systems of reactiondiffusion equations," International Journal of Nonlinear Sciences and Numerical Simulation, vol. 7, no. 4, pp. 411-418, 2006.

[7] M. Gorji, D. D. Ganji, and S. Soleimani, "New application of He's homotopy perturbation method," International Journal of Nonlinear Sciences and Numerical Simulation, vol. 8, no. 3, pp. 319-328, 2007.

[8] Y. R. Shi, X. J. Xu, Z. X. Wu et al., "Application of the homotopy analysis method to solving nonlinear evolution equations," Acta Physica Sinica, vol. 55, no. 4, pp. 1555-1560, 2006.

[9] J. Q. Mo and J. S. Yao, "Homotopic mapping method for the solution of soliton to a perturbed KdV equation," Acta Physica Sinica, vol. 57, no. 12, pp. 7419-7422, 2008.

[10] L. F. Shi and J. Q. Mo, "Soliton-like homotopic approximate analytic solution for a disturbed nonlinear evolution equation," Acta Physica Sinica, vol. 58, no. 12, pp. 8123-8126, 2009.

[11] S. Liao, "Comparison between the homotopy analysis method and homotopy perturbation method," Applied Mathematics and Computation, vol. 169, no. 2, pp. 1186-1194, 2005.

[12] Y. R. Shi and H. J. Yang, "Application of a homotopy analysis method to solving a dissipative system," Acta Physica Sinica, vol. 59, no. 1, pp. 67-74, 2010.

[13] J.H. He, "Homotopy perturbation method for solving boundary value problems," Physics Letters A, vol. 350, no. 1-2, pp. 87-88, 2006.

[14] G. Adomian, "A review of the decomposition method in applied mathematics," Journal of Mathematical Analysis and Applications, vol. 135, no. 2, pp. 501-544, 1988.

[15] G. Adomian, Nonlinear Stochastic Systems and Applications to Physics, Kluwer Academic Publishers Group, Dordrecht, The Netherlands, 1989.

[16] S. Abbasbandy, "A numerical solution of Blasius equation by Adomian's decomposition method and comparison with homotopy perturbation method," Chaos, Solitons and Fractals, vol. 31, no. 1, pp. 257-260, 2007.

[17] S. Abbasbandy and M. T. Darvishi, "A numerical solution of Burgers' equation by modified Adomian method," Applied Mathematics and Computation, vol. 163, no. 3, pp. 1265-1272, 2005.

[18] M. A. Malkov, "Spatial chaos in weakly dispersive and viscous media: a nonperturbative theory of the driven KdV-Burgers equation," Physica D: Nonlinear Phenomena, vol. 95, no. 1, pp. 62-80, 1996.

[19] K. Y. Guan and G. Gao, "Qualitative analysis and traveling wave solutions of the Burgers-KdV equation," Science in China A, vol. 30, no. 1, pp. 64-73, 1987.

[20] S. D. Liu and S. S. Liu, "KdV-Burgers equation modelling of turbulence," Science in China A, vol. 34, no. 9, pp. 938-945, 1991.
[21] B. Hong, "New Jacobi elliptic functions solutions for the variable-coefficient MKdV equation," Applied Mathematics and Computation, vol. 215, no. 8, pp. 2908-2913, 2009.

[22] S. Liao, Beyond Perturbation: Introduction to the Homotopy Analysis Method, CRC Press, New York, NY, USA, 2004. 


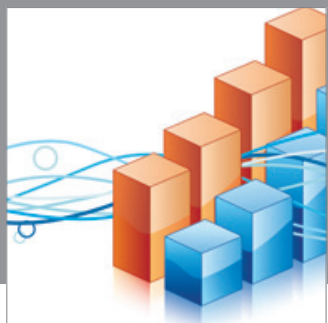

Advances in

Operations Research

mansans

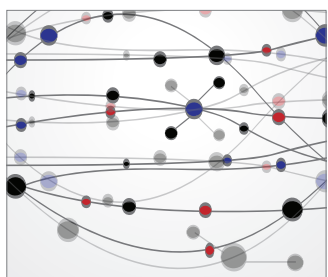

The Scientific World Journal
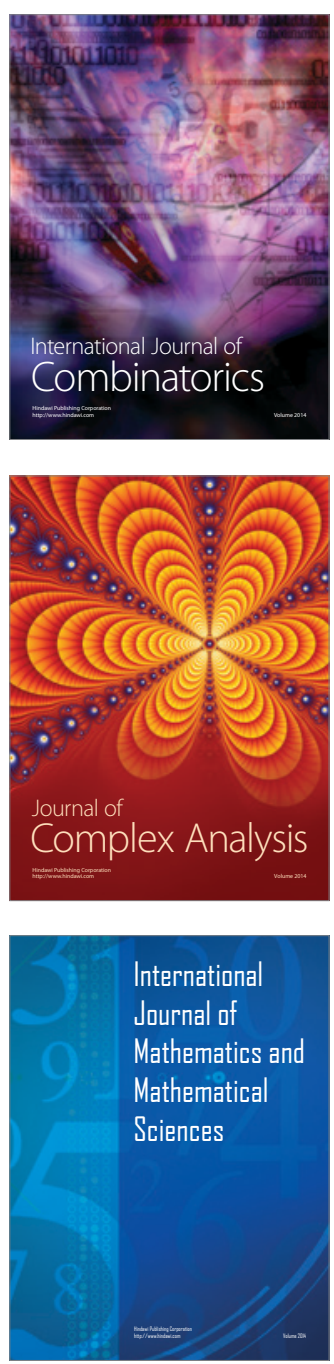
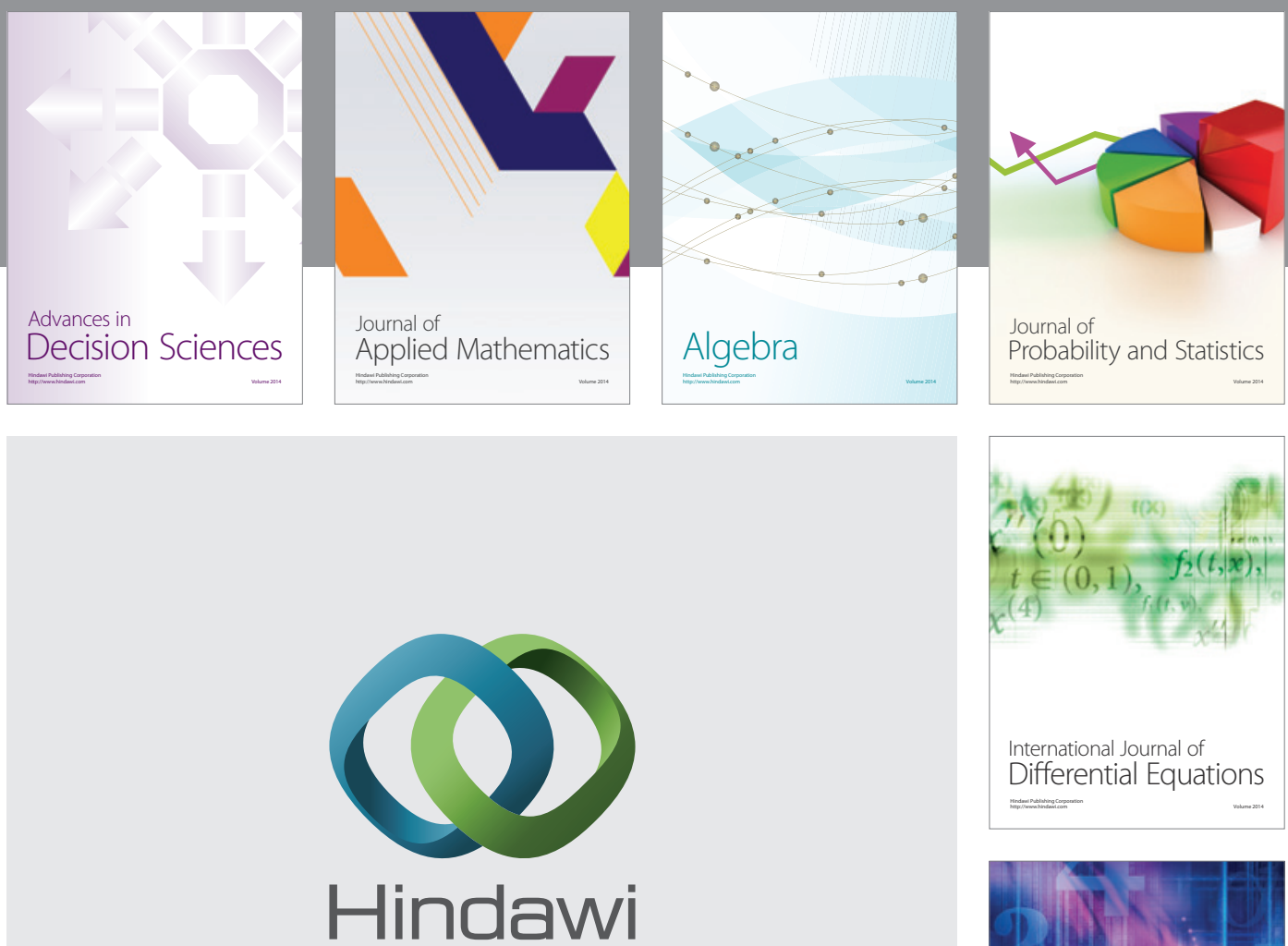

Submit your manuscripts at http://www.hindawi.com
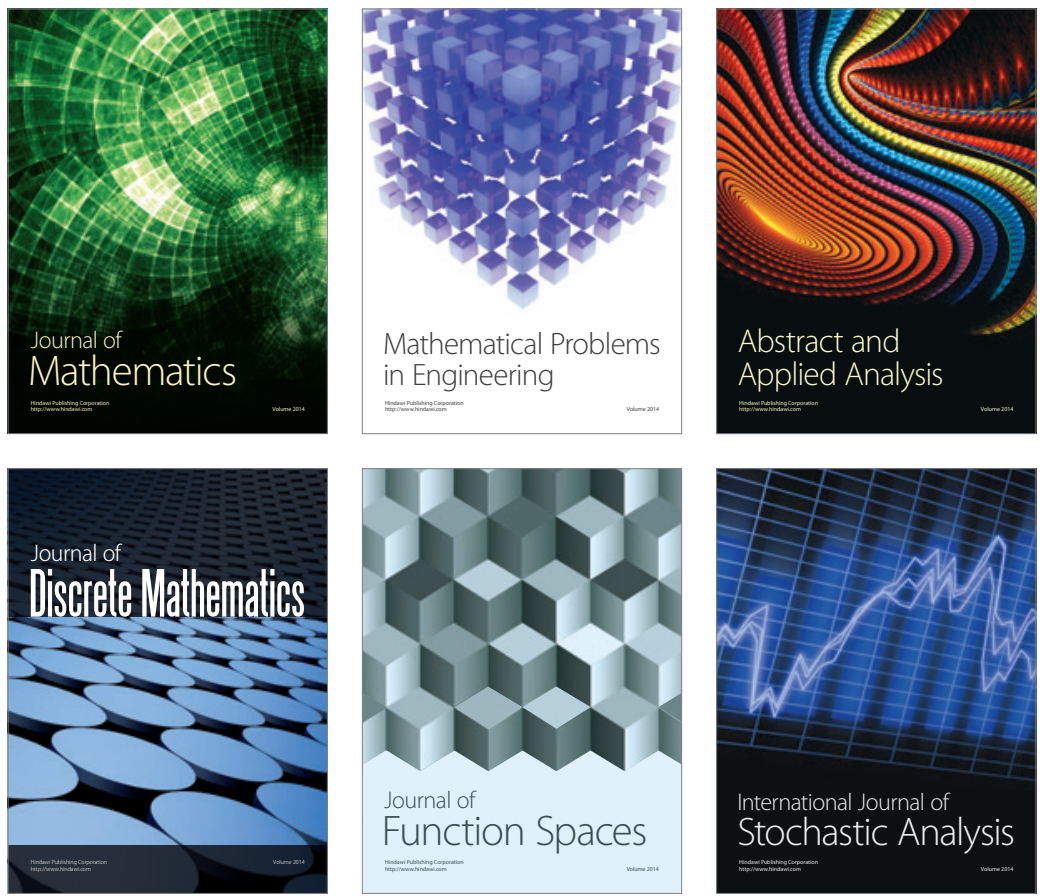

Journal of

Function Spaces

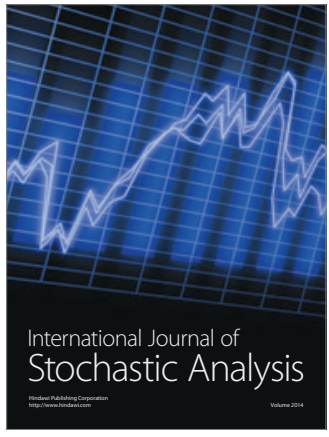

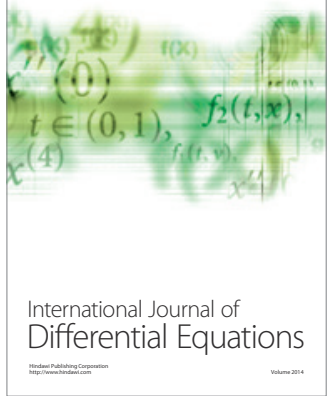
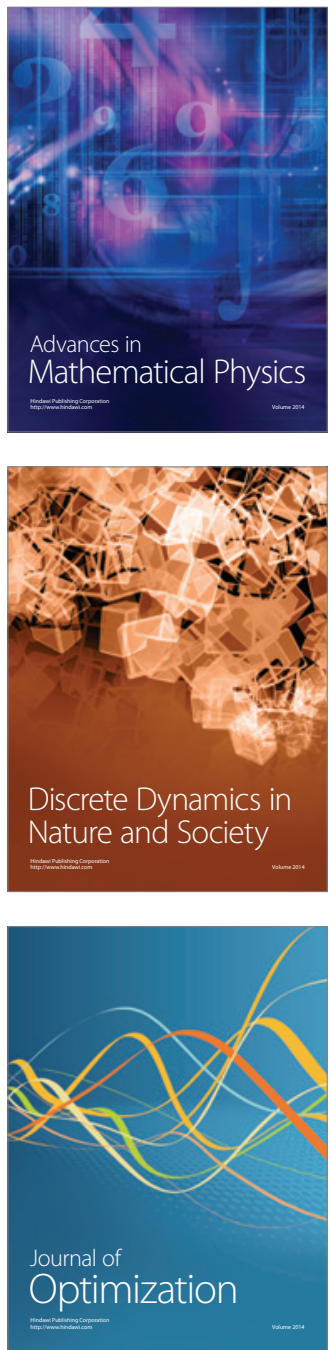